Itinéraires Itinéraires

Littérature, textes, cultures

2013-1 | 2013

La fiction aujourd'hui

\title{
Hélène Cixous
}

Le récit comme tissage de la vie et de l'écriture

\section{Maxime Decout}

\section{OpenEdition}

\section{Journals}

Édition électronique

URL : http://journals.openedition.org/itineraires/848

DOI : 10.4000/itineraires.848

ISSN : 2427-920X

Éditeur

Pléiade

\section{Édition imprimée}

Date de publication : 1 octobre 2013

Pagination : 121-137

ISBN : 978-2-343-01791-4

ISSN : 2100-1340

Référence électronique

Maxime Decout, « Hélène Cixous », Itinéraires [En ligne], 2013-1 | 2013, mis en ligne le 19 septembre 2014, consulté le 01 mai 2019. URL : http://journals.openedition.org/itineraires/848; DOI : 10.4000/ itineraires.848

\section{(c) (i) (9)}

Itinéraires est mis à disposition selon les termes de la licence Creative Commons Attribution - Pas d'Utilisation Commerciale - Pas de Modification 4.0 International. 


\section{Hélène Cixous}

\section{Le récit comme tissage de la vie et de l'écriture}

\section{Résumé}

L'œuvre d'Hélène Cixous témoigne d'un désir constant de mêler la diction à la fiction tout en récusant le paradigme mimétique. Dans cette perspective, l'entre-deux entre fiction et diction autorise une lucidité sur l'existence du récit, renforcée par un métatextuel abondant. Les textes refusent que la fiction joue de l'illusion référentielle et qu'elle masque la présence du récit. De la sorte c'est un autre réel qui se définit, un réel non pas considéré comme séparé de et antérieur à la sphère littéraire mais bien comme une expérience narrative, comme constitué lui-même de récits.

Mots clés : Cixous, récit, fiction, diction, métatextuel

\section{Abstract}

The work of Hélène Cixous exhibits a constant desire to mix diction with fiction, yet challenges the mimetic paradigm. With this in mind, the interface between fiction and diction elucidates the presence of the narrative. Abundant metatextuality heightens this existence. In these texts, fiction cannot play with an illusion of reality, nor can it mask the narrative. Subsequently, another reality that is tied to literature in the present, and is considered an experience that is itself composed of the narrative, is defined.

Keywords: Cixous, narrative, fiction, diction, metatextual

L'œuvre d'Hélène Cixous se constitue autour d'un noyau d'inspiration autobiographique évident, avec ses personnages récurrents que sont le père défunt, Georges Cixous, ou la mère, Ève Klein. Mais au cœur de ce discours, se déploie un imaginaire singulier qui laisse libre cours à la fiction. La vie personnelle s'ouvre aux pulsions de l'imaginaire et du symbolique, déployant un discours mythique et allégorique qui empêche toute identification stricte d'un matériau autobiographique comme d'une fiction réaliste et mimétique. Tout semble alors concourir à un mélange de la diction et de la fiction, comme si la vie personnelle ne pouvait être 
approchée qu'à travers le recours au fictionnel. Et pourtant, paradoxalement, dans de nombreux textes, cette fiction reste menacée. L'écriture référentielle, l'illusion de personnages en tant que personnes, la diégèse logique et cohérente sont déboutées. Chez Cixous, le personnage devient souvent une sorte de figure ou un ensemble de pulsions, de paroles. Aussi, si la diction ne peut se passer de la fiction, cette dernière n'existe jamais sans une certaine lucidité à son égard ${ }^{1}$. C'est pourquoi la fiction chez Cixous se pense souvent en lien avec un discours métatextuel prégnant qui la questionne en questionnant le récit. De la sorte, entre diction, fiction et récit se joue un nouage étroit qui propose une profonde modification de l'appréhension de la chose littéraire.

Et ce renouveau du récit semble avoir une source essentielle : Hélène Cixous est romancière et critique littéraire. Forte de cette double filiation, elle ménage toujours dans ses récits une véritable conscience critique vis-àvis de la fiction. Aussi, afin d'intégrer souplement la réflexion métatextuelle dans les romans, qui n'est pas pour autant une forme de nombrilisme littéraire mais bien une interrogation plus vaste sur l'être et le monde, ses romans font du Récit en lui-même l'un des protagonistes du texte, aux côtés de ces autres instances aujourd'hui problématiques que sont l'auteur ou le sujet. Cette exigence absolue de lucidité sur ce qu'est un récit est le point de départ d'un questionnement sur l'événement du récit en tant qu'expérience qui s'inscrit au-delà du littéraire stricto sensu. C'est depuis cette insistance sur la métatextualité que la romancière trace sa voie propre, en marge d'autres voies féminines contemporaines, comme celles de Sophie Calle, d'Annie Ernaux ou de Christine Angot qui questionnent elles aussi les liens entre le vécu et la fiction. Si la dépersonnalisation est l'un des éléments de son travail sur le sujet comme chez Annie Ernaux, elle refuse pourtant le minimalisme de l'écriture ou la suppression du « je » comme on le trouve dans Les Années par exemple. C'est plus visiblement de Doubrovsky que Cixous semble se rapprocher. Car c'est au sein du cadre générique commun qu'est l'autofiction et avec une écriture baroque et jubilatoire, que les deux écrivains ménagent une place première à une métatextualité contagieuse, qui porte autant sur le texte que sur ses divers intertextes. Dans Fils comme dans les œuvres de Cixous, un mouvement de flux et de reflux s'organise entre la fictionnalisation du commentaire, souvent inséré dans l'intrigue, et l'interprétation de la fiction, invitant à interroger l'ensemble des fils et des filiations herméneutiques qui se tissent, comme le propose la polysémie du titre choisi par Doubrovsky. Et ce jusqu'à considérer autant le texte comme fils du métatexte que le métatexte comme fils du texte. Ainsi pensée, la fiction préside à un brouillage des catégories, proposant de reconsidérer

1. «Récit » est employé ici dans le sens que lui donne Gérard Genette dans Figures III, Paris, Le Seuil, coll. «Poétique », 1972, p. 72. Il désigne l'énoncé narratif qui assume la relation des événements, en opposition avec l'histoire ou diégèse, qui est le contenu, et la narration qui est l'acte de raconter les événements. 
l'écriture et la lecture comme un ensemble inextricable où le lecteur et l'auteur sont des herméneutes de tous les textes-tissus, que ce soit celui du monde ou des livres, qui s'intriquent sans solution de continuité.

\section{La folittérature : quand la fiction devient la clef de la diction}

Les récits d'Hélène Cixous s'appuient pour la plupart sur une volonté nette d'exhiber les dispositifs mis en place pour narrer l'histoire, si bien que le lecteur ne peut jamais oublier que le récit existe et qu'il est raconté. L'illusion d'une histoire autonome, soit simplement racontée par un narrateur homodiégétique unique et cohérent, constitué en tant qu'illusion de personne, soit narrée de façon extérieure de sorte que c'est alors la fiction elle-même qui se constitue en illusion de réel, est impossible dans des récits tels que Messie, La Fiancée juive, ou Neutre. Mais la fiction disparaît-elle pour autant? Car de nombreux textes d'Hélène Cixous relèvent assurément d'un discours factuel, d'apparence autobiographique, comme $O R$, Le jour où je n'étais pas là, Si près, Ève s'évade. Une part importante de l'œuvre semble s'écrire comme une sorte de livre unique et continué, sans cesse repris, avec les personnages principaux issus de la vie de la romancière que sont sa mère, son père, son fils mort et sa grand-mère Omi. Néanmoins, les interférences avec l'autofiction sont nombreuses, comme si se mêlaient toujours, à divers degrés, autobiographie et fiction, comme dans Le jour où je n'étais pas là. Il s'agit ainsi d'élaborer une sorte de fiction personnelle, de mythe individuel qui structure l'œuvre à l'échelle d'ensemble par sa récurrence et sa reprise. D'autres œuvres, au contraire, appartiennent plus visiblement au récit fictionnel comme Messie, La Fiancée juive, Tombe ou Neutre. Mais que faire de textes délibérément incertains comme Jours de l'an où la diégèse s'absente partiellement, perd en cohérence, au profit d'une sorte de réflexion personnelle, narrée par un je? Le statut de Jours de l'an pose ainsi problème, utilisant autant un pacte autobiographique, ce pacte défini par Philippe Lejeune, qu'un pacte fictionnel.

Cette incertitude entre fiction et diction, telle que Gérard Genette les a analysées ${ }^{2}$, semble magistralement exhibée par l'ouverture d'Osnabrück. Le récit s'apparente à une autobiographie, narrée à la première personne, dans un texte où la cohérence diégétique est de mise. Le pacte autobiographique est explicite dès les premières pages avec la mention du nom de la mère de la narratrice, Ève Klein, devenue, par son mariage, Ėve Cixous ${ }^{3}$. Pourtant, c'est la mort de la mère qui est narrée. Ce n'est qu'ensuite que le récit dénonce cette entrée en matière, cette diction première, comme une fiction ${ }^{4}$. Le mort n'était pas le bon : c'est le père qui a disparu. La suite du récit rétablit la vérité autobiographique. Pourquoi cette substitution

2. Gérard Genette, Fiction et diction [1979], Paris, Le Seuil, coll. «Points essais », 2004.

3. Hélène Cixous, Osnabrück, Paris, Des femmes, 1999, p. 16.

4. Ibid., p. 28. 
initiale? Parce que c'est la perte que la narratrice a perdue, c'est le souvenir de la mort du père qui s'est évaporé, si bien qu'elle « n'a pas pu raconter [s]on histoire sans [s]'y tromper ${ }^{5} »$. Mais d'une perte à l'autre, de celle de la mère à celle du père, d'une fiction à une diction, semble aussi se dire l'impossibilité d'un discours autobiographique pur, seul, nu. Comme si la fiction restait l'impulsion nécessaire, le point de départ obligé pour un dire sur soi. C'est bien l'aveu de la fonction vitale de la fiction qui se dit, vitale parce qu'elle seule pourra dire la mort et la perte, vitale parce qu'elle seule pourra indiquer la pluralité irréductible de la vie et des identités.

Par conséquent, c'est la dimension fictionnelle de la diction qui est régulièrement soulignée, comme dans Le Livre de Promethea : "Je ne redoute rien autant que l'autobiographie. L'autobiographie n'existe pas. Mais tant de gens croient que cela existe. Alors je déclare ici solennellement : l'autobiographie n'est qu'un genre littéraire ${ }^{6}$. » La narratrice affirme ici que toute écriture de soi est un dispositif littéraire comme tel, sans qu'existe une véritable différence avec d'autres formes plus volontiers fictionnelles. Ces propos ne sont pourtant pas une fin de non-recevoir définitive pour la dimension autobiographique, qui ne cesse de croître au fil du temps chez Cixous. Mais, si l'autobiographie pure n'existe pas, c'est parce que l'univers de Cixous est un univers de récits et de textes dont le réel ne peut se séparer. La vie puise toujours dans une fascination violente pour la littérature. C'est pourquoi, une large part de la diction, délaissant toute attention à la dimension factuelle et à la véracité des événements, tient en l'aveu d'une certaine relation avec le monde du livre et la fiction. Sans prétendre à la reconstitution d'une vie, avec un regard rétrospectif explicatif et unificateur, la diction se forme par touches éparses, dans une dissémination permanente qui met l'accent sur des sensations, sentiments et pensées, ou sur quelques épisodes fragmentaires dont la signification se trouve accrue. Pour rendre compte de ce lien singulier entretenu avec la fiction, Hélène Cixous le met en œuvre, le met à l'œuvre : c'est la fiction qui sera le moyen de dire la relation personnelle établie avec la fiction. Le récit se conçoit alors comme le lieu fou de l'entre-deux : «Provisoirement je nomme ce livre Le Récit. L'histoire que devrait raconter Le Récit pourrait tenir dans l'envergure de deux mots : folie littérature et plus exactement dans l'étreinte d'un seul : folittérature ${ }^{7}$. »

\section{Ibid.}

6. Hélène Cixous, Le Livre de Promethea, Paris, Gallimard, 1983, p. 27-28. On mesurera ici la foncière différence avec le travail d'Annie Ernaux et son constant refus de la fiction comme de l'autofiction, au profit d'une autobiographie dépersonnalisée. Voir par exemple Annie Ernaux, « Je n'ai rien à voir avec l'autofiction », entretien avec Christine Ferniot et Philippe Delaroche, Lire, no 362 , février 2008, p. 84-99.

7. Hélène Cixous, Manhattan, lettres de la préhistoire, Paris, Galilée, coll. «Lignes fictives », 2002, p. 38. 
Cette « folittérature » est celle qui étreint fiction, diction et récit. Car Manhattan raconte une histoire difficile à situer entre fiction et diction. L'imagination, à travers le " cas de contagion par la littérature ${ }^{8}$ " qui est rapporté, vient brouiller le passé, le remodeler. Il s'agit de l'histoire amoureuse de la narratrice avec un homme, rencontré dans la Bibliothèque métropolitaine de New York, qui se fait passer pour mourant et appeler Gregor. Celui-ci se construit une identité qui entre en résonance avec la vie de Cixous, puisqu'il lui rappelle son père mort, Georges, et avec la littérature, par le biais de son apparition dans la bibliothèque et le fait qu'il est un être presque échappé d'une fiction de Kafka. Le personnage semble ainsi le creuset où diction et fiction vont fusionner. Gregor possède en effet un " caractère anormalement littéraire ${ }^{9}$ ». Il s'est inventé une identité fictionnelle basée sur les plus grands récits, si bien qu'il se réduit à « un assemblage-montage fabuleux de citations et de références empruntées à la littérature mondiale ». Ce personnage est l'illustration même du pouvoir dangereux de la fiction telle que Cixous le décrit dans sa pratique passionnelle et pulsionnelle de la lecture, notamment avec Montaigne ${ }^{10}$. Mais il indique aussi que la fiction est nécessaire, en tant qu'échappée de soi et reconstruction du réel. La diction seule demeure impossible. Elle est une sorte de chimère inaccessible. Danger autant que salut, ce bovarysme devient chez Cixous le seul moyen pour vivre et le seul moyen pour écrire. Gregor apparaît comme l'emblème même de la fiction et de la déformation que le récit fait subir à la vie personnelle. La façon dont sa mise en scène est décryptée par la narratrice est métaphorique de la manière dont le récit met lui-même à distance la fiction sans pour autant l'invalider. Si l'illusion de réel de la fiction est donc souvent menacée chez Cixous, le sentiment d'une diction n'est parfois pas mieux assuré. D'autant que, si la fiction est bien, par le mensonge qu'elle autorise, une pluralisation du sens et un questionnement de l'identité, elle ne peut pas entièrement disparaître. Si la romancière refuse en effet, notamment dans ses premiers textes, la mimésis traditionnelle, c'est grâce à la fiction qu'elle procède. Car la fiction, rappelle Paul Ricœur, est l'affirmation du pouvoir de nier le monde, de suspendre la référence en vue d'une recréation ${ }^{11}$.

On comprend mieux alors le fonctionnement singulier d'OR, les lettres de mon père où la diction sur le père, parce que diction sur un vide et une absence, ne pourra se constituer que depuis la fiction, à la manière dont Georges Perec procédait par exemple dans $W$ ou le Souvenir d'enfance où seul le jeu de suture entre les parties fictionnelles et autobiographiques permettait de tenir un discours sur la disparition de la mère. $\mathrm{Si}$, comme chez
8. Ibid.
9. Ibid., p. 85 .
10. Ibid., p. 82.
11. Paul Ricœur, La Métaphore vive, Paris, Le Seuil, coll. « L'ordre philosophique », 1975, p. 310 . 
Perec, la fiction et la diction restent partiellement sectorisées dans Osnabrück, celles-ci se nouent et s'intriquent absolument dans $O R$, au point que l'on ne peut plus les séparer. Il semble donc qu'Hélène Cixous, à des degrés plus ou moins forts en fonction des textes, tente la difficile conciliation de la fiction et de la diction, répondant à cette tendance que repère Dominique Rabaté : « Entre une impossible diction et une fiction insuffisante (c'est-à-dire qui ne saurait plus exister par elle-même), la littérature contemporaine invente une formule instable, un mixte problématique ${ }^{12}$. » Cette formule instable, ce mixte problématique, Hélène Cixous le baptise « folittérature ».

\section{Le tissage de la diction dans la fiction : vers le chant du récit}

Entre fiction et diction, c'est le récit qui travaille à établir des passages et des communications. Une zone particulièrement sensible à ces échanges se trouve dans la figure de l'auteur, que plusieurs œuvres de la romancière interrogent. Point de jonction entre l'intérieur et l'extérieur du livre, passerelle inévitable entre la vie et l'œuvre, l'auteur est à même d'articuler la fiction et la diction au sein du récit. Dans Double oubli de l'orang-outang, Edgar Allan Poe, modèle pour la romancière, est celui qui sait « copier la réalité afin de la faire passer pour la vérité de la fiction. Afin de faire passer la vérité de la réalité à la fiction ${ }^{13} \gg$. Faire passer : il y a là comme un mot talisman qui pourrait désigner les rapports problématiques de la fiction et de la diction. Faire passer : c'est-à-dire autant faire prendre la réalité pour et la faire pénétrer à l'intérieur de. Deux opérations qu'il s'agit de distinguer; la première tient à l'illusion de réalité, la deuxième à la mise en récit. Cixous raconte ainsi que Charles Meyron a exposé ses doutes à Baudelaire sur la réalité de l'auteur Edgar $\mathrm{Poe}^{14}$. Baudelaire lui répond que «cet Edgar Poe a de la réalité ${ }^{15} »$, même s'il ne l'a jamais vu. D'où l'hypothèse qu'Edgar Poe soit « le fantôme d'un artiste capable de s'inventer lui-même comme il invente ses personnages, tous également dotés d'une véridicité hors du commun ${ }^{16}$ ». " Il pourrait en ce cas être une fiction nécessitée et engendrée par les puissances de la fiction. » Si l'auteur n'existe plus en tant que personne mais se construit en même temps que sa fiction, de manière nécessaire, toute tentative de diction se ferait ensuite depuis un auteur qui a lui-même été façonné par sa fiction. Si bien que toute diction se verrait nécessairement estampillée d'une dimension fictionnelle. D'où la question que Cixous se pose face à son œuvre qui met en question sa vie, qui met en doute

12. Dominique Rabaté, «L'entre-deux: fictions du sujet, fonctions du récit», dans Frontières de la fiction, [En ligne], www.fabula.org/forum/colloque99.php.

13. Hélène Cixous, Double oubli de l'orang-outang, Paris, Galilée, 2010, p. 192.

14. À ce titre, l'intertexte chez Cixous est souvent ce qui « montre la scène littéraire, c'est-àdire le texte en ses constructions figurales », comme le rappelle Mireille Calle-Gruber dans Du café à l'éternité. Hélène Cixous à l'œuvre, Paris, Galilée, 2002, p. 16.

15. Hélène Cixous, Double oubli de l'orang-outang, op. cit., p. 192.

16. Ibid., p. 194. 
la diction et la réalité de la personne, par le biais du processus de fiction : « Suis-je une fiction $?{ }^{17}$ » L'univers entier a basculé du côté de la fiction :

Nous « vivons », si cela s'appelle encore « vie », en ligne, des vies qui ne sont pas les nôtres et qui nous font circuler impuissants dans des histoires dont nous n'avons pas la moindre idée en réalité. Et nous ne pourrons plus jamais utiliser l'expression «en réalité »en réalité $e^{18}$.

En réalité : un syntagme tabou, une expression devenue impossible à prononcer, presque excommuniée, parce que toute réalité est nourrie de fiction, comme le montre aussi le bovarysme de Manhattan. Ce que Cixous affirme ici explicitement, elle ne cesse de le mettre à l'œuvre dans ses textes : le refus des illusions mimétiques, qu'elles soient du côté de la diction ou de la fiction, au profit d'une conscience pleine que tout est récit.

Le «tissage de la vie dans l'écriture ${ }^{19}$ », c'est par la fiction en ellemême que Cixous se propose de le questionner. Ce tissu, dans son appellation même, évoque déjà la solidarité presque indissociable de la vie et du textus du récit. Un livre comme Jours de l'an est évidemment nourri d'une dimension personnelle affichée, tout en cédant régulièrement la place à une réflexion sur le récit qui utilise les voies de la fiction. Il propose une fiction interrogative qui se focalise notamment sur le clivage entre le Moi et l'auteur, sans cesse réaffirmé par le dialogue de ces deux figures, dont aucune ne paraît responsable de l'ensemble du livre. Cixous semble écrire à rebours de la « mort de l'auteur » et pourtant avec elle. L'auteur devient un transfuge qui passe de l'univers de la diction à celui de la fiction puisqu'il est décrit comme une sorte de personnage, aussi incertain que les autres. Appelé « l'auteur », sans caractérisation sociale ou psychologique, il ne se définit que par son statut auctorial. Sa seule détermination est d'ordre sexuel puisque les adjectifs, déterminants ou pronoms qui gravitent autour de lui sont féminins. S'esquisse ainsi un lien ténu avec la figure de la narratrice, des personnages principaux souvent féminins, mais aussi d'Hélène Cixous, réalisant une fusion de la diction et de la fiction ${ }^{20}$. Mais ce lien, à peine suggéré, est troublé à plusieurs reprises, empêchant toute approche inspirée de Sainte-Beuve :

Pourquoi parlé-je de l'auteur comme si elle n'était pas moi? Parce qu'elle n'est pas moi. Elle part de moi et va où je ne veux pas aller. Souvent je sens qu'elle est mon ennemie. [...] Parfois nous ne faisons qu'une presque ${ }^{21}$.

17. Ibid., p. 195.

18. Ibid., p. 200.

19. Ibid., p. 12.

20. On consultera avec profit les réflexions d'Eberhard Gruber, «L'analytique troublée par la fiction », dans Mireille Calle-Gruber et Marie Odile Germain (dir.), Genèses, généalogies, genres : autour de l'œuvre d'Hélène Cixous, Paris, Galilée, 2006, p. 65-81, qui proposent une analyse de la question du sujet chez Hélène Cixous selon une tout autre perspective.

21. Hélène Cixous, Jours de l'an, Paris, Des femmes, 1990, p. 153. 
Entre symbiose et scission, l'auteur est une possibilité du Moi, une sorte de pulsion textuelle qui accomplit sa structure langagière. Si le Moi est en discordance avec l'auteur, c'est bien sur cette déshérence que le récit se construit aussi comme processus d'entre-deux entre fiction et diction. De ce fait, même le je de la narratrice n'occupe pas de façon absolue une position de surplomb par rapport à la narration. Lui et l'auteur, dépris de leurs prérogatives usuelles, s'inscrivent dans le livre à côté de personnages et d'un récit qui leur échappent. Ce renversement paradoxal de la maîtrise de la fiction fait bien que, dans Jours de l'an, « l'auteur s'est sentie écrite, frappée d'écriture 22 ». C'est l'autonomie du récit qui s'affiche, la force d'une parole qui fraye sa propre trace en s'affranchissant de tous les carcans.

Dès lors, la narratrice, dans un renversement des hiérarchies établies, délègue par endroits la parole à l'auteur, permettant l'insertion d'une deuxième narration : «j'ai passé une journée avec Melchior, raconte l'auteur ${ }^{23} \gg$. Melchior, cet étrange roi mage, est celui qui conduit l'auteur vers le chant du récit. En effet, l'auteur conte ici l'histoire étrange et paradigmatique de toute l'œuvre, de toute œuvre peut-être. Dans ce mythe des origines du récit, le personnage qui la guide, Melchior, lui échappe. Celui-ci « avait pris le corps de l'auteur pour nager, grimper, nicher l'espace et avec les deux corps faire un corps humain ${ }^{24} \gg$. Le récit se pense à travers ce qui pourrait être une sorte d'androgyne textuel, inscrit dans la logique de l'érotisme du texte propre à Cixous. Rejoignant donc l'auteur sans pour autant fusionner avec elle, le personnage rejoue l'aventure d'Ulysse telle que la lit Maurice Blanchot en tant que paradigme du récit. En effet, le récit naîtrait du chant des Sirènes lorsqu'Ulysse accepte d'aller jusqu'à ce chant, lorsqu'il accepte de devenir Homère ${ }^{25}$ : c'est-à-dire lorsque le personnage touche et rejoint l'auteur, que la fiction vient atteindre la diction, et que le chant fait événement en soi au lieu d'être simple production d'un être conscient et maître de sa fiction. La fusion rêvée de Melchior et de l'auteur désignerait ainsi un point originel où la parole s'affranchit et se fait récit. C'est pourquoi Melchior affirme : « Je suis un personnage libre [...]. On ne peut pas me demander de jouer ce rôle-ci ou cet autre rôle ${ }^{26}$. »

Aussi, dans cette aventure mythique, Melchior devient-il le cicérone de l'auteur. Il la conduit vers « une grotte fermée que l'auteur n'a pas vue ${ }^{27}$ » et que celle-ci ne découvre que grâce à lui : « Je ne suis pas un personnage libre, parce que je suis sans le royaume de Melchior. Et je n'arrive à être un peu voyante que dans certains chapitres de mes livres. Raconte-moi

22. Ibid., p. 64.

23. Ibid., p. 77.

24. Ibid.

25. Maurice Blanchot, Le Livre à venir [1959], Paris, Gallimard, coll. «Folio essais », 1986, p. 14-15.

26. Hélène Cixous, Jours de l'an, op. cit., p. 77.

27. Ibid. 
encore ce que tu vois, a dit l'auteur ${ }^{28}$. » L'auteur, à la différence du personnage, n'est pas libre. Elle est un personnage sous la dépendance du personnage. Sa fiction ne provient pas entièrement d'elle mais se crée à partir de l'univers fictionnel qui révèle son autonomie. Dans ce mythe des origines fictionnelles, c'est toute la mythologie de la création qui se réinvente, en se fondant sur une liberté paradoxale des personnages, plus à même de voir que l'auteur, plongé dans une cécité certaine et une forte incapacité à écrire. Le cœur vivant de la parole du récit, le chant des Sirènes en sa pureté originelle, est bien cet instant de l'entre-deux entre fiction et diction où se réalise la disparition de celui qui s'en est approché pour que seul demeure le chant, nu, vide de toutes compromissions. Le tissage de la vie dans l'écriture, l'intrication fiction-diction, est bien au cœur même de la définition du récit.

\section{Le Récit : un récit du récit}

Le lien étroit entre fiction et diction, au cœur de la définition même du récit et de son chant autonome, fait ainsi que le récit raconte ses propres origines. La fiction dit le récit et sa naissance, affirme que le texte est récit. L'incipit de Double oubli de l'orang-outang, emblématique de cette démarche, s'ouvre en se retournant sur l'univers du récit : " "Je l'aurai donc relu". J'écrivis aussitôt cette phrase. Comme on note une prophétie ${ }^{29}$. » Le début installe immédiatement le texte sous le double signe de la relecture et de l'écriture. Scripteur et lecteur, le je est confronté à un événement proprement clos dans le monde du récit. Ce début, qui n'en est pas un, repose sur une situation d'énonciation tue qui vient laisser la phrase initiale hors de toute amarre. Comme si cette phrase contenait sa propre situation d'énonciation, était à elle-même sa propre situation d'énonciation : celle qui appartient au monde du récit et non à un réel extérieur. Le futur antérieur qui ouvre le texte assure bien de l'inéluctable du récit qui fait « Événement ${ }^{30}$ » en soi, qui vient faire événement dans l'événement qu'est déjà l'incipit du récit ${ }^{31}$. Car la narratrice a en fait découvert un carton qui contient l'ancien manuscrit d'un de ses livres : dès à présent, elle est tout entière happée par le récit et le récit qu'elle fait de ce récit : «J'étais toute à la phrase maintenant. » Tout le récit sera alors la consignation du « rapport de l'Événement ${ }^{32} »$, celui de l'apparition du carton, du surgissement du texte, au sein même de l'événement de ce récit. Cette structure d'emboîtement empêche

28. Ibid., p. 78.

29. Hélène Cixous, Double oubli de l'orang-outang, op. cit., p. 11.

30. Ibid.

31. Voir à ce sujet les analyses de Jacques Derrida, «H.C. pour la vie, c'est à dire... », dans Mireille Calle-Gruber (dir.), Hélène Cixous, croisées d'une æeuvre (actes du colloque de Cerisy), Paris, Galilée, p. 38. Derrida voit justement l'œuvre de Cixous comme une «poétique de l'événement», «de la puissance de l'événement» et de «l'événement en puissance $»$.

32. Ibid., p. 20. 
de penser un récit clos et une fiction mimétique détachée du récit. Ce qui fait la fiction n'est pas la retranscription d'une réalité mais bien l'événement du récit : 1'approche du chant des Sirènes selon Blanchot. La fiction est désignée comme la boîte noire à même d'enregistrer l'événement que constitue le récit.

Par conséquent, si Blanchot fait d'Ulysse le rusé celui qui jouit « du spectacle des Sirènes sans risques et sans en accepter les conséquences ${ }^{33}$ "», se tournant ensuite vers le pis-aller du roman qui est la narration logique et cohérente de la suite des épisodes constituant son voyage, Hélène Cixous, elle, n'écrit pas de romans. Elle accepte le risque d'aller vers le chant des Sirènes et de produire un récit. Car son récit répond à la définition qu'en donne Blanchot : «Le récit est héroïquement et prétentieusement le récit d'un seul épisode, celui de la rencontre d'Ulysse et du chant insuffisant et attirant des Sirènes ${ }^{34}$. \ C'est-à-dire ce récit exemplaire de Jours de l'an où Melchior, le je et l'auteur rencontrent le chant, l'événement du chant en lui-même. Le roman, incarné dans la pensée de Blanchot par Ulysse, est un horizon toujours présent mais impossible à atteindre pour le récit :

\footnotetext{
J'envie la structure si clairement ordonnée de L'Odyssée : pour chaque localité, un épisode, et inversement. Un vrai trajet marin. [...] Le plus étrange c'est lorsque, rejeté, fracassé, démâté, échoué sur une côte, le récit, quand enfin il revient à lui, se retrouve sur le même rocher où il avait fait escale trente ans plus tôt. [...] L'illusion tient alors la réalité solidement ${ }^{35}$.
}

L'épopée homérique apparaît comme une entité structurante qui autorise un contrôle du réel par la fiction. Certes, il y a dans L'Odyssée une pulsion régulière où les épisodes « sont happés par une force d'attraction monstrueuse » qui menace finalement la structure, mais toujours, tout revient à sa place. La fiction, avec ses «épisodes », est menacée par une force déstructurante et obscure qui, chez Cixous, devient centrale : c'est celle du Récit. C'est pourquoi Cixous avoue n'avoir « jamais désiré écrire un "roman", avec des personnages archiromanesques, des exaltés, des souvenirs descendants d'un délire qui pousse râpeux » : " Quelle imitation naïve, dangereuse. Et l'on ne sait même pas que l'on est dans l'imitation $^{36}$. » La lucidité convoquée par Cixous concerne la capacité qu'a le récit de plonger le lecteur dans l'imitation, jusqu'à oublier l'existence fondatrice du récit. Le récit propose donc un autre mode d'organisation du texte ainsi qu'un autre rapport de la fiction au réel. Il se pense comme une « nuée d'interrogations errantes ${ }^{37}$ ». Sa logique opère sans synthèse organisatrice.

33. Maurice Blanchot, op. cit., p. 11.

34. Ibid., p. 13.

35. Hélène Cixous, Double oubli de l'orang-outang, op. cit., p. 60-61.

36. Ibid., p. 199.

37. Ibid., p. 202. 


\section{L'effet de vitre ou la lamelle de verre isophone entre le récit et la fiction}

Afin de mettre à distance l'illusion référentielle, Cixous travaille de façon récurrente et variée à scinder l'univers de la fiction de celui du récit, séparant l'histoire de la narration qui en est faite. Dans Manhattan, elle avoue :

Cette remarque est totalement extérieure à l'histoire. Elle appartient au Récit. Et encore au récit version 2000.

Avant cette date une lamelle de verre isophone se glissait entre l'histoire et le Récit ${ }^{38}$.

Cette séparation provient, dans le cadre de ce texte, de la difficulté à dire le passé et à retrouver la diction, que celle-ci soit réelle ou qu'elle soit fictive mais présentée comme réelle dans le cadre de la diégèse. Les strates du récit de cette histoire, accumulées au cours du temps, témoignent de son inadéquation à ce qui a été vécu : «D'innombrables mues du Récit datant d'époques plus ou moins anciennes gisent dans La Chemise jaune ${ }^{39}$. $» \mathrm{La}$ fiction-diction qui s'élabore est ainsi progressive. Le récit se construit peu à peu, déformant et reformant le vécu. C'est pourquoi le texte propose au lecteur la liste des différents incipits envisagés au cours du temps pour narrer la fabula ${ }^{40}$. Le récit ne parvient pas à commencer, incapable qu'il est de cerner l'histoire. Et ces esquisses de débuts ne sont pas placées à l'ouverture du livre, comme si plusieurs commencements s'offraient au lecteur. L'ensemble de ces procédés permet de placer l'histoire narrée à un degré second. De fait, ce qui est narré au premier degré n'est plus la fiction mais la relation entretenue avec cette fiction et avec sa mise en récit. La diégèse principale est donc celle où s'élabore le récit d'une fabula secondaire à laquelle le lecteur n'a accès qu'indirectement, qu'en passant par la médiation d'une réflexion sur le récit. La fiction est éloignée à un deuxième degré, montrant que l'on n'y accède que par le biais d'un récit : ce processus met en valeur le récit, empêche l'autonomie de la fiction, oblige le lecteur à ne pas oublier l'étape de la mise en récit, à ne pas oublier que ce qu'il lit est un récit. Cixous se montre finalement incapable d'écrire l'histoire première : « Je n'écrirai pas ce livre ${ }^{41} »$, affirme-t-elle. Le livre sera le récit de l'impossibilité d'écrire directement l'histoire première (qu'elle soit fiction ou diction). Le récit semble ne plus naître que de l'échec à narrer directement la fiction : il se fait récit de la mise en récit de la fiction et de son échec.

La vitre intercalée en permanence entre la fiction et le récit permet alors au récit de se constituer au-delà de la continuité supposée d'une diégèse. Aussi est-ce un processus permanent de découpe qui joue afin de

38. Hélène Cixous, Manhattan, op. cit., p. 43.

39. Ibid., p. 51.

40. Ibid., p. 53-56.

41. Ibid., p. 57. 
briser toute linéarité et toute autonomie de la fiction. La rencontre avec Gregor, ce personnage qui "fait passer » le réel au fictionnel et «fait passer $\gg$ le fictionnel pour le réel, est l'objet central impossible à raconter; elle se morcelle. Relatée au milieu du texte, elle n'offre pas l'illusion d'une histoire cohérente puisque ses divers événements sont scindés sous forme d'une liste d'épisodes numérotés ${ }^{42}$. Complétant ce jeu de fragmentation, un regard scrupuleusement proche de celui de l'analyste est convoqué dans le texte, afin d'exhiber les éléments qui, dans la fiction, désignent le récit. L'analyse par la narratrice d'un épisode s'effectue méthodiquement, selon un ensemble de détails sélectionnés et interprétés en six tirets qui mettent l'accent sur la démarche analytique et viennent rompre la continuité de l'histoire. Il s'agit du «[d]étail [...] agrandi à la dimension du Tout ${ }^{43} »$. Ainsi le couvre-œil de Gregor, élément de la fiction, devient un signifiant qui désigne le processus du récit, « la Caméra du Récit ». Hélène Cixous procède ici à rebours de la mise en intrigue telle que Ricœur l'analyse ${ }^{44}$ : elle ne livre pas au lecteur l'intégralité pure de la fiction mais, avant celleci, en extrait des détails signifiants, des métonymies qui sont liées autant à la fiction qu'au récit. Ce qui est donné à lire est donc déjà une lecture à l'avance de la fiction. Ce passage est d'ailleurs surdéterminé quant à l'isotopie de la découpe. Outre les tirets, les éléments d'interprétation sélectionnés métaphorisent cette opération : un couvre-œil, un demi-écureuil, une gravure, une cicatrice et deux lanternes magiques qui projettent, en les découpant, des scènes sur un mur. On voit bien comment le signifié de ces détails renvoie autant à la fiction qu'aux modalités de sa mise en récit, et que le récit se constitue finalement au-delà de l'histoire proprement dite. Manhattan instaure de la sorte une distance quasi systématique entre le récit et la fiction, qui se marque notamment par la réflexion métatextuelle :

\footnotetext{
Dans Le Récit il y aurait à ce moment-là un gros plan sur le paquet de livres que j'allais lire dans la semaine.

(cette histoire est en extérieurs été brûlant pour la première partie. La seconde partie : tout entière en intérieurs glacés, ténébreux, neiges, tempêtes) ${ }^{45}$.
}

Deux phénomènes jouent ensemble : l'exhibition de la fictionnalité du texte, brisant l'illusion de réel, ainsi que la non-concordance du temps du récit et du temps de la fiction; l'histoire n'est jamais racontée directement, c'est la temporalité du récit, de la réflexion sur la fiction que le lecteur suit. Comme chez Diderot ou Sterne, mais sans la désinvolture qui les caractérise, c'est bien l'histoire racontée, la fabula, qui est la cible des interventions métatextuelles. Elle se fragmente et semble impossible à transmettre d'un seul tenant.

42. Ibid., p. 86-87.

43. Ibid., p. 48.

44. Voir Paul Ricœur, Temps et récit I [1983], Paris, Le Seuil, coll. « Points essais », 1991.

45. Hélène Cixous, Manhattan, op. cit., respectivement p. 119 et 145. 


\section{L'aventure métatextuelle}

L'œuvre de Cixous est ainsi, dans son ensemble, le récit de l'aventure du texte, par le biais du métatextuel, que l'on peut définir avec Bernard Magné comme l'ensemble des moyens dont un texte dispose " pour assurer dans son corps même la désignation de tout ou partie de ses mécanismes constitutifs ${ }^{46} »$. Neutre est à ce titre une tentative extrême : la fiction n'existe plus que pour être l'aventure du Récit et de ses différents éléments. Ce sont les entités qui composent le Récit qui ont remplacé les personnages, comme le montre notamment la liste inaugurale qui ouvre le texte, en lieu et place de la présentation des personnages au début d'une pièce de théâtre. Cette liste, appelée « Joueurs, pièces et revenants ${ }^{47}$ », comprend le Récit, le Texte, le Sujet... La présence massive du paratexte, à travers cette liste initiale, une série d'exergues et un ensemble de notes, vient compléter ce dispositif de sortie de l'illusion d'une fiction autonome et véridique. La fiction de Neutre se veut explicitement le résultat de la construction d'un récit. Neutre est un véritable essai sur le récit, mais un essai qui emprunte les voies de la fiction. C'est la fiction qui est chargée de parler du Récit et de mettre en scène ses aventures. Le métatextuel devient ici la règle générale du textuel.

Pour autant, si certains textes, comme Neutre, tendent à empêcher partiellement la fabula de se constituer, le métatextuel ne demeure pas vraiment un facteur d'opposition à la fiction. En effet, si celui-ci met en un certain sens à distance la fiction mimétique, il exhibe la mise en récit et donc une fiction, mais détachée du prédicat mimétique. C'est pourquoi l'interruption de l'adhésion à l'univers fictionnel, par le métatextuel, réaffirme cependant l'existence incontestable de la fiction. On voit ainsi que la réflexion sur le récit peut s'intégrer dans la fiction sans la faire disparaître. Cette fiction devient le creuset où le récit se fabrique et se questionne, le lieu d'une plongée archéologique dans le récit et l'écriture, une véritable fouille, comme celle que réalise la narratrice dans le carton de Double oubli de l'orang-outang. Ce dernier texte souligne en effet la complémentarité du métatextuel et de la fabula, puisque l'histoire se focalise sur un manuscrit retrouvé et devient par là même un questionnement sur le récit. La fiction est bien l'expression d'un rapport avec un livre, d'une relation singulière avec le récit et la fiction. Si la perception de la fiction est bien conditionnée par la relation établie avec le lecteur ${ }^{48}$, alors il semble que le métatextuel participe de cette construction et des rapports du lecteur avec la fiction. Le lecteur chez Cixous se trouve placé dans une position de négociation des différents actes issus de la fiction, de la diction et du métatextuel. D'autant

46. Bernard Magné, « Métatextuel et lisibilité », Protée, vol. XIV, nº 1-2, printemps 1986, p. 77.

47. Hélène Cixous, Neutre [1972], Paris, Des femmes, 1998, p. 17.

48. Voir les analyses de Kendall Walton, Mimesis as Make-Believe, Cambridge, Harvard University Press, 1990. 
que toute fiction, sans autre procédé, tend à faire naître une interrogation du lecteur sur les processus mis en œuvre, et donc sur le récit. Dans cette perspective, il ne convient donc pas d'opposer la fiction, avec sa fabula, et le métatextuel. Car la fiction, à un degré moindre, concourt finalement au même effet que le métatextuel. C'est bien ce qui autorise Cixous, beaucoup plus que le Nouveau Roman, à les utiliser conjointement.

Tombe réalise cette fusion du métatextuel et de la fiction. Au début du récit, la fiction conserve les pleins pouvoirs; le texte se présente comme une sorte de mythe de l'amour et de la mort, recourant à des personnages peu caractérisés, soustraits à toute dimension sociale, dans un décor souvent incertain, à travers une diégèse peu suivie. Le texte procède par fragments et pulsions, sans suivre une chronologie rationnelle. Il narre l'aventure de Dioniris, personnage mythique nouveau, empruntant ses traits à Adonis, Dionysos comme Achille. C'est l'amour avec la narratrice, elle aussi incertaine et non caractérisée, qui est chanté. Cet amour, comme dans une réécriture virgilienne, tourne autour du Sexe d'or et des noces des amants. Et pourtant ce mythe est aussi un mythe de l'écriture. La fiction incorpore le monde du récit et narre conjointement l'aventure des personnages et l'aventure de l'écriture. En effet, sans cesse, l'histoire mythique qui est racontée est décrite comme un récit: «Ce serait l'histoire dont la périphérie serait le temps et chaque phrase un des mondes ${ }^{49}$. » $\mathrm{C}^{\prime}$ 'est à une véritable textualisation de la fiction que le livre appelle. Pour imaginer un jeune homme «nouveau, saillant», le texte donne par exemple ce conseil : " Pour cela, imagine une écriture couchée d'abord et ordinaire sur le papier mais cette même écriture ensuite levée ensuite lavée ensuite délivrée grimpante multipliée ${ }^{50}$. » La fusion des deux univers fait que les éléments du monde de l'écrit diffusent dans celui de la fiction et participent eux aussi de l'écriture du mythe. C'est même à travers les catégories de l'écrit que les éléments de la fiction sont saisis et pensés :

je n'aurai ni repos ni virgule.

Il y avait un texte du cœur qui ne cessait d'inscrire son silence sans le rompre sans repos sans virgule sans vie.

faisant du monde notre lit et de notre lit la grande page repliée de notre connaissance.

nous nous y roulions de rire [...], sur le livre sans prêter garde à la syntaxe, moi phrase en fuite, sans sujet même.

Nous composions un texte insondable.

Lui le stylo moi l'encre ${ }^{51}$.

L'écriture est une aventure amoureuse comme l'amour et la sexualité sont une aventure textuelle. La vie des amants est alors décrite comme une

49. Hélène Cixous, Tombe [1973], Paris, Le Seuil, coll. « Réflexion », 2008, p. 37.

50. Ibid., p. 53-54.

51. Ibid., respectivement p. 55, 84, 88 et 145 . 
syntaxe libérée, sans points ni virgules ${ }^{52}$, à la manière même dont le texte est écrit : "Je nous trouvais semblables à deux syntagmes venimeux liés mais tendus à distance par une copule comme dans la phrase Dioniris / est / ici que je me répétais en m'endormant ${ }^{53} \gg$. Aussi une véritable synergie de la fiction et du récit s'organise-t-elle si bien que la quête mythique du Sexe d'or acquiert son répondant textuel à travers la «Virgule d'or ${ }^{54}$ ». C'est bien un érotisme textuel qui naît, de sorte que l'engendrement du texte est décrit comme un engendrement sexuel ${ }^{55}$. Ces noces des univers de la fiction et du récit vont jusqu'à l'apparition de mots-valises qui apparient les deux mondes au cœur d'un syntagme unique résumant le processus de cristallisation dont le texte est l'opérateur : le « litvre », « l'amour-texte », le «Textamant ${ }^{56} »$. Il s'agit bien d'une autre forme du tissage de la vie dans l'écriture et de l'écriture dans la vie. Le métatextuel dénotatif ${ }^{57}$, pourtant senti comme antagoniste de la fabula, ne vient pas l'hypothéquer. Au contraire, les deux fabulae, celle de l'histoire et celle du Récit, s'accouplent intimement, faisant de l'aventure du métatextuel l'aventure de la fiction ${ }^{58}$.

Si l'œuvre de Cixous expose donc bien l'aventure d'une écriture, il s'agit de voir que les procédés et les enjeux ne peuvent toutefois être ramenés de façon unique à ceux du Nouveau Roman. D'abord, dans les œuvres les plus récentes de Cixous, la transmission de la fabula est nettement moins compromise qu'avec le Nouveau Roman ou qu'avec Neutre. Ensuite, le métatextuel qu'utilisent les textes est majoritairement dénotatif, ce qui peut être le cas avec le Nouveau Roman, mais qui n'est pas une règle générale. L'autocommentaire se fait donc volontairement explicite empêchant toute séparation entre l'univers de la fiction et le récit, ce qui relève de toute évidence d'une autre conception du réel. C'est pourquoi Hélène Cixous ne joue pas de la capture d'un récit par un autre, comme dans Projet pour une révolution à New York par exemple, où le lecteur prend sans cesse ce qu'il lit pour le récit avant que celui-ci lui soit désigné comme récit dans le récit. Le Nouveau Roman, à cet égard, multiplie les passages et les surprises, il fomente l'incertain et crée l'inquiétude et l'ambivalence, par le relais de la mise en abyme ${ }^{59}$. La métalepse, telle que la pratique Borges

52. Ibid., p. 101.

53. Ibid., p. 108.

54. Ibid., p. 194.

55. Ibid., p. 191-192.

56. Ibid., respectivement p. 145, 193 et 95.

57. Pour une analyse de la distinction entre métatextuels connotatif et dénotatif, voir Bernard Magné, op. cit.

58. À titre de comparaison, on pourra songer à la pratique oulipienne du métatextuel, comme chez Perec avec La Disparition, ou Roubaud avec La Belle Hortense. Le métatextuel y est souvent connotatif afin de concilier la réflexion littéraire et le plaisir de la fiction, sans les opposer comme c'est parfois le cas dans le Nouveau Roman.

59. Voir par exemple la manière dont Dällenbach explore les spécificités de la mise en abyme dans le Nouveau Roman dans Le Récit spéculaire, Paris, Le Seuil, 1977. 
notamment, concourt, dans cette perspective, à un effet relativement proche. Mais ces jeux sont hors de propos avec Cixous, où l'introduction du récit dans l'univers de la fiction ne vise pas l'étrange ou le fantastique. Le lecteur est averti dès le départ que la fiction et le récit ne sont plus dissociables et que cette anastomose ne relève aucunement d'une opération inquiétante ou métaphorique. Si Cixous n'utilise pas de métalepses, c'est bien parce que, chez elle, comme dans Jours de l'an, l'auteur ne peut pas venir franchir la limite entre le monde du livre et la diégèse : celle-ci n'existe tout simplement plus. Le récit est totalement incorporé dans la fiction. Il ne s'agit pas de son inclusion momentanée, de jeux d'échos ou de références métaphoriques ponctuelles, même si celles-ci peuvent être présentes comme dans Tombe avec l'évocation de « tissus ${ }^{60}$ » où s'imprime l'histoire des doigts de la narratrice, ou les tentatives de «nouer » et « dénouer » effectuées en lien avec le «tapis de vie ». Ce qui se réalise est autre : il s'agit d'une unité totale. Les textes de Cixous créent le sentiment que l'écriture et le récit appartiennent aussi au monde et à la vie, sans distinction possible. Il ne s'agit donc pas comme dans le Nouveau Roman de rendre la fiction métaphorique de l'écriture et du récit mais de faire de la fiction le lieu de l'aventure du récit comme l'on peut en faire le lieu de l'aventure, non pas métaphorique mais directement signifiée, de personnages. Chez Hélène Cixous, fiction et récit, comme réalité et récit, constituent deux modalités d'une même chose, l'avers et le revers d'une seule médaille.

Il y a donc, entre fiction et récit, une relation presque sexuelle - et non pas conflictuelle, comme dans certains textes de Robbe-Grillet qui visent à faire de l'œuvre une entité autonome qui s'affranchit de toute réalité extralinguistique en supprimant l'histoire, cette notion balzacienne périmée. Puisque Neutre, Tombe ou Jours de l'an ne cessent de se représenter, de représenter leurs éléments et leurs processus, ce métatextuel vient mettre à mal la représentation mimétique du monde, comme dans le Nouveau Roman, mais pas l'existence de la fiction, contrairement au Nouveau Roman. La narration autoreprésentée indique ainsi une chose essentielle : le monde représenté dans les œuvres n'est pas le décalque d'un réel objectif mais la retranscription d'un réel déjà vécu sur le mode de la fiction et de la narration. C'est ainsi la réflexion métatextuelle, où se dit l'aventure d'un récit, qui devient l'un des moteurs essentiels de la fiction, un moteur presque unique dans Neutre, alors que sa dynamique est partagée avec une histoire dans Jours de l'an, Tombe ou Manhattan. Le mouvement de l'œuvre épouse ainsi le mouvement de la réflexion métatextuelle sur le récit.

Les trois strates qui président au texte chez Cixous, la diction, la fiction et le récit, ne peuvent donc être comprises en les sectorisant. Les rapports entre fiction et diction sont de complémentarités, puisqu'une diction sans fiction devient impensable. Si l'univers littéraire d'Hélène Cixous est bien

60. Hélène Cixous, Tombe, op. cit., p. 41-42. 
constitué d'un matériau premier issu de sa vie, celui-ci s'avère indissociable de la fiction qui le porte et qui le transmue. Bio et graphein ne sont pas simplement posés dans une relation opérationnelle qui partirait de la vie pour la retranscrire par l'écriture. La fiction et la diction se nouent, se tissent, et c'est ce tissu qui forme le véritable textus. Le récit chez Cixous ne peut donc se définir que dans cet entre-deux. Aussi, puisqu'une diction, qui est nécessairement une illusion mimétique, est impossible, la fiction qui vient la soutenir, ne peut pas elle non plus s'engouffrer dans le paradigme traditionnel du mimétique. Les textes de Cixous se veulent ainsi un constant rappel de l'existence du récit et de la fiction en tant que tels. C'est bien pourquoi ils placent la fiction dans un deuxième degré par rapport au récit qui en vient à constituer la matière première de l'œuvre. La fiction lue n'est plus une histoire diégétique mimétique mais une fiction sur la création du récit. La généralisation des procédés métatextuels souligne l'existence du récit, sans pour autant nier la fiction. Dans une complémentarité nouvelle, diction, fiction et métatextuel jouent ensemble, en soulignant la mise en récit spontanée que tout un chacun fait de l'expérience, c'est-à-dire cette « narrativité inchoative qui ne procède pas de la projection, comme on dit, de la littérature sur la vie, mais qui constitue une authentique demande de récit », comme le rappelle Paul Ricœur ${ }^{61}$. Si bien que l'œuvre ne prétend jamais dire un réel objectif qui aurait une existence autonome en dehors de nous mais le réel tel qu'il nous parvient, toujours médiatisé par notre faculté à le mettre en récit.

Maxime Decout Université Charles-de-Gaulle-Lille 3 